\title{
Targeted Metabolomics Reveals Birth Screening Biomarkers for Biliary Atresia in Dried Blood Spots
}

Yongtao Xiao 1,2,3,\#,* PhD, Ying Zhou ${ }^{1, \#}$ MD, PhD, Kejun Zhou 4,\#, PhD, Wei Cai $1,2,3,{ }^{*} \mathrm{MD}, \mathrm{PhD}$

${ }^{1}$ Department of Pediatric Surgery, Xin Hua Hospital, School of Medicine, Shanghai Jiao Tong University, Shanghai 200092, China;

${ }^{2}$ Shanghai Institute of Pediatric Research, Shanghai 200092, China;

3 Shanghai Key Laboratory of Pediatric Gastroenterology and Nutrition, Shanghai 200092, China;

${ }^{4}$ Human Metabolomics Institute, Inc., Shenzhen 518109, Guangdong, China

Correspondence to:

Yongtao Xiao, Ph.D.

Division of Pediatric Gastroenterology and Nutrition, Xin Hua Hospital,

Shanghai Jiao Tong University

No. 1665, Kong Jiang Road, Shanghai 200092, China

Tel: 86-21-25076445; Fax: 86-21-65791316

E-mail: xiaoyongtao@xinhuamed.com.cn

Wei Cai, MD, Ph.D.

Department of Pediatric Surgery, Xin Hua Hospital,

Shanghai Jiao Tong University

No. 1665, Kong Jiang Road, Shanghai 200092, China

Tel: 86-21-25076441; Fax: 86-21-65791316

E-mail: caiw204@sjtu.edu.cn

\# These authors contribute this work equally.

\section{Table of Contents}

Table S1. Demographic information of the newborns enrolled. Page S-2

Table S2. A total of 24 metabolites were differentially expressed between the BA and Control infants in the discovery cohort. Page S-5

Table S3. A total of 27 metabolites were differentially expressed between the BA and Control infants in the validation cohort. Page S-6

Table S4. Four most significantly altered canonical pathways between BA and comparison infants. Page S-7

Table S5. The AUC, cutoff, sensitivity and specificity value of single altered metabolites. Page S-8

Table S6. The AUC, cutoff, sensitivity and specificity value of altered metabolites combinations. Page S-9 
Table S1. Demographic information of the newborns enrolled.

\begin{tabular}{|c|c|c|c|c|}
\hline Cohort & Group & gender & $\begin{array}{c}\text { Gestational age } \\
\text { (week + day) }\end{array}$ & $\begin{array}{c}\text { Birth weight } \\
\text { (g) }\end{array}$ \\
\hline Discovery & Control 1 & Female & $36+1$ & 3030 \\
\hline Discovery & Control 2 & Female & 39 & 3450 \\
\hline Discovery & Control 3 & Male & 41 & 3900 \\
\hline Discovery & Control 4 & Male & $38+5$ & 3335 \\
\hline Discovery & Control 5 & Female & $35+4$ & 2295 \\
\hline Discovery & Control 6 & Female & $35+3$ & 2355 \\
\hline Discovery & Control 7 & Male & $40+5$ & 3500 \\
\hline Discovery & Control 8 & Female & $39+4$ & 3580 \\
\hline Discovery & Control 9 & Male & $40+2$ & 3340 \\
\hline Discovery & Control 10 & Female & $38+1$ & 3400 \\
\hline Discovery & Control 11 & Male & $37+3$ & 3500 \\
\hline Discovery & Control 12 & Female & $40+5$ & 3500 \\
\hline Discovery & Control 13 & Female & $39+1$ & 3400 \\
\hline Discovery & Control 14 & Male & $38+1$ & 3400 \\
\hline Discovery & Control 15 & Male & 39 & 3650 \\
\hline Discovery & Control 16 & Male & $39+5$ & 4000 \\
\hline Discovery & Control 17 & Male & $38+6$ & 3350 \\
\hline Discovery & Control 18 & Male & $39+6$ & 3550 \\
\hline Discovery & Control 19 & Male & 41 & 3800 \\
\hline Discovery & Control 20 & Male & 32 & 1930 \\
\hline Discovery & Control 21 & Female & $35+1$ & 2105 \\
\hline Discovery & Control 22 & Male & $34+5$ & 3295 \\
\hline Discovery & Control 23 & Male & $38+2$ & 3180 \\
\hline Discovery & Control 24 & Male & 41 & 3445 \\
\hline Discovery & Control 25 & Male & $39+4$ & 3020 \\
\hline Discovery & Control 26 & Female & $39+2$ & 3635 \\
\hline Discovery & Control 27 & Male & 35 & 2250 \\
\hline Discovery & Control 28 & Male & 35 & 1805 \\
\hline Discovery & Control 29 & Female & $35+4$ & 2875 \\
\hline Discovery & Control 30 & Female & $34+5$ & 2345 \\
\hline Discovery & Control 31 & Male & 37 & 3440 \\
\hline Discovery & Control 32 & Female & $33+3$ & 1790 \\
\hline Discovery & Control 33 & Male & $35+3$ & 2185 \\
\hline Discovery & Control 34 & Female & $39+3$ & 2905 \\
\hline Discovery & Control 35 & Female & 40 & 3400 \\
\hline Discovery & Control 36 & Female & $37+5$ & 2800 \\
\hline Discovery & Control 37 & Female & $39+4$ & 3860 \\
\hline Discovery & Control 38 & Female & 34 & 2135 \\
\hline Discovery & Control 39 & Male & $39+1$ & 3260 \\
\hline
\end{tabular}




\begin{tabular}{|c|c|c|c|c|}
\hline Discovery & Control 40 & Female & $37+5$ & 2920 \\
\hline Discovery & BA 1 & Female & 395 & 3730 \\
\hline Discovery & BA 2 & Female & 38 & 3350 \\
\hline Discovery & BA 3 & Male & $41+2$ & 3480 \\
\hline Discovery & BA 4 & Female & $39+3$ & 3900 \\
\hline Discovery & BA 5 & Female & $38+2$ & 3360 \\
\hline Discovery & BA 6 & Female & $41+3$ & 3200 \\
\hline Discovery & BA 7 & Male & 36 & 2145 \\
\hline Discovery & BA 8 & Female & $39+4$ & 2880 \\
\hline Discovery & BA 9 & Male & $41+4$ & 4225 \\
\hline Discovery & BA 10 & Female & 41 & 2970 \\
\hline Discovery & BA 11 & Female & $40+4$ & 3360 \\
\hline Discovery & BA 12 & Female & 36.1 & 3030 \\
\hline Discovery & BA 13 & Male & $40+5$ & 3500 \\
\hline Validation & Control 1 & Female & $40+1$ & 3390 \\
\hline Validation & Control 2 & Male & $40+4$ & 3330 \\
\hline Validation & Control 3 & Female & $39+3$ & 3300 \\
\hline Validation & Control 4 & Male & $37+6$ & 2600 \\
\hline Validation & Control 5 & Male & 34 & 2140 \\
\hline Validation & Control 6 & Male & $40+2$ & 3590 \\
\hline Validation & Control 7 & Male & $39+4$ & 3260 \\
\hline Validation & Control 8 & Female & $39+5$ & 4070 \\
\hline Validation & Control 9 & Male & $36+1$ & 3520 \\
\hline Validation & Control 10 & Female & $40+1$ & 3180 \\
\hline Validation & Control 11 & Male & $39+3$ & 3540 \\
\hline Validation & Control 12 & Male & $38+3$ & 3410 \\
\hline Validation & Control 13 & Female & $39+3$ & 3450 \\
\hline Validation & Control 14 & Male & $39 / 2$ & 3460 \\
\hline Validation & Control 15 & Female & $41+1$ & 3310 \\
\hline Validation & Control 16 & Female & $40+2$ & 3660 \\
\hline Validation & Control 17 & Female & $39+3$ & 3030 \\
\hline Validation & Control 18 & Male & $40+3$ & 3620 \\
\hline Validation & Control 19 & Male & $35+3$ & 2680 \\
\hline Validation & Control 20 & Female & $38+5$ & 3400 \\
\hline Validation & Control 21 & Female & $38+4$ & 3050 \\
\hline Validation & Control 22 & Male & $40+2$ & 3450 \\
\hline Validation & Control 23 & Male & $39+4$ & 3430 \\
\hline Validation & Control 24 & Male & $37+6$ & 2890 \\
\hline Validation & Control 25 & Male & $38+3$ & 2980 \\
\hline Validation & Control 26 & Male & $36+2$ & 2200 \\
\hline Validation & Control 27 & Female & 38 & 2290 \\
\hline Validation & Control 28 & Female & $41+1$ & 3020 \\
\hline Validation & Control 29 & Male & 41 & 3600 \\
\hline
\end{tabular}




\begin{tabular}{|c|c|c|c|c|}
\hline Validation & Control 30 & Male & $40+5$ & 3570 \\
\hline Validation & Control 31 & Male & $40+2$ & 3745 \\
\hline Validation & Control 32 & Male & $40+6$ & 3630 \\
\hline Validation & Control 33 & Female & $39+3$ & 3150 \\
\hline Validation & Control 34 & Male & $39+2$ & 3335 \\
\hline Validation & Control 35 & Male & $39+6$ & 3290 \\
\hline Validation & Control 36 & Male & $38+5$ & 3690 \\
\hline Validation & Control 37 & Female & $38+5$ & 2845 \\
\hline Validation & Control 38 & Male & $39+3$ & 3740 \\
\hline Validation & Control 39 & Female & $39+6$ & 3445 \\
\hline Validation & Control 40 & Female & $39+2$ & 3235 \\
\hline Validation & Control 41 & Female & $36+4$ & 3700 \\
\hline Validation & Control 42 & Female & $38+5$ & 2820 \\
\hline Validation & Control 43 & Male & $40+2$ & 3345 \\
\hline Validation & Control 44 & Female & $39+1$ & 3880 \\
\hline Validation & Control 45 & Female & $38+5$ & 3370 \\
\hline Validation & Control 46 & Female & $38+2$ & 3310 \\
\hline Validation & Control 47 & Male & 38 & 2830 \\
\hline Validation & Control 48 & Female & $39+6$ & 3470 \\
\hline Validation & Control 49 & Male & $40+4$ & 3750 \\
\hline Validation & Control 50 & Male & $39+3$ & 4020 \\
\hline Validation & Control 51 & Male & $403 / 7$ & 3350 \\
\hline Validation & Control 52 & Female & 39 & 2820 \\
\hline Validation & Control 53 & Female & $40+3$ & 3180 \\
\hline Validation & Control 54 & Male & $41+1$ & 4070 \\
\hline Validation & Control 55 & Male & $38+4$ & 2900 \\
\hline Validation & Control 56 & Male & $41+1$ & 3300 \\
\hline Validation & Control 57 & Male & $36+6$ & 3640 \\
\hline Validation & Control 58 & Female & $40+2$ & 3260 \\
\hline Validation & Control 59 & Female & 40 & 3460 \\
\hline Validation & Control 60 & Male & 41 & 3350 \\
\hline Validation & BA 1 & Male & 38.5 & 3335 \\
\hline Validation & BA 2 & Female & $39+4$ & 3580 \\
\hline Validation & BA 3 & Female & 39 & 3450 \\
\hline Validation & BA 4 & Female & $35+4$ & 2355 \\
\hline Validation & BA 5 & Male & $40+2$ & 3340 \\
\hline Validation & BA 6 & Male & $39+4$ & 3020 \\
\hline Validation & BA 7 & Female & $40+2$ & 3660 \\
\hline Validation & BA 8 & Female & 39 & 3270 \\
\hline
\end{tabular}


Table S2. A total of 24 metabolites were differentially expressed between BA and Control in the discovery cohort.

\begin{tabular}{|c|c|c|c|}
\hline Metabolite & Uni_P & FC & OPLSDA_VIP \\
\hline THCA & $3.97 \mathrm{E}-08$ & 0.195756 & 2.26158 \\
\hline Glutamic acid & $3.10 \mathrm{E}-05$ & 0.637256 & 2.347589 \\
\hline Ketoleucine & 0.000125 & 1.961411 & 2.108749 \\
\hline Glycine & 0.000505 & 0.734613 & 2.401933 \\
\hline GCA & 0.000604 & 0.352054 & 2.324192 \\
\hline TCA & 0.00066 & 0.426005 & 2.606182 \\
\hline 3-methyl-2-oxopentanoic acid & 0.000934 & 1.530509 & 2.07057 \\
\hline 2-hydroxyglutaric acid & 0.002113 & 0.723272 & 2.471095 \\
\hline alpha-Linolenic acid & 0.002113 & 1.797277 & 1.576722 \\
\hline Serine & 0.003098 & 0.761187 & 2.191116 \\
\hline Creatine & 0.004072 & 0.741377 & 2.490009 \\
\hline $\mathrm{HCA}$ & 0.004464 & 1.88164 & 1.197166 \\
\hline Maltotriose & 0.005518 & 1.550227 & 1.490855 \\
\hline alpha-Aminobutyric acid & 0.006336 & 0.795135 & 1.49139 \\
\hline N-Acetylserine & 0.009459 & 0.766302 & 1.144401 \\
\hline Palmitoylcarnitine & 0.011279 & 0.759944 & 1.426042 \\
\hline Acetylglycine & 0.011468 & 0.619919 & 1.525027 \\
\hline alpha-Ketoisovaleric acid & 0.012344 & 3.898872 & 1.577486 \\
\hline Maltose/Lactose & 0.013834 & 1.388729 & 1.320945 \\
\hline Glutaconic acid & 0.015427 & 0.792083 & 2.241869 \\
\hline Adipoylcarnitine & 0.019842 & 1.243292 & 1.253431 \\
\hline Indoleacetic acid & 0.02115 & 1.451276 & 1.144745 \\
\hline Gluconolactone & 0.027883 & 0.887236 & 1.646343 \\
\hline 2-hydroxy-3-methylbutyric acid & 0.02793 & 0.698165 & 1.159318 \\
\hline
\end{tabular}


Table S3. A total of 27 metabolites were differentially expressed between BA and Control in the validation cohort.

\begin{tabular}{|c|c|c|c|}
\hline Metabolite & Uni_P & FC & OPLSDA_VIP \\
\hline Glyceric acid & 4.10E-05 & 0.330984 & 2.087314 \\
\hline alpha-Ketoisovaleric acid & 0.000107 & 2.115741 & 1.804438 \\
\hline Indoleacetic acid & 0.000113 & 2.065611 & 1.835051 \\
\hline Histidine & 0.000214 & 5.845786 & 1.758531 \\
\hline AMP & 0.000248 & 0.550592 & 2.162536 \\
\hline Ketoleucine & 0.00051 & 1.879592 & 1.538674 \\
\hline Glutamic acid & 0.001025 & 0.607639 & 2.22202 \\
\hline Valeric acid & 0.001431 & 0.493705 & 2.019191 \\
\hline Acetylcarnitine & 0.001528 & 3.046936 & 1.29252 \\
\hline Propionylcarnitine & 0.001631 & 2.769829 & 1.168952 \\
\hline GCA & 0.00225 & 0.130435 & 2.057441 \\
\hline Cinnamic acid & 0.002397 & 0.405132 & 1.888498 \\
\hline Butyric acid & 0.002553 & 0.343684 & 1.668279 \\
\hline Citramalic acid & 0.002718 & 0.788407 & 1.645075 \\
\hline 2-hydroxyglutaric acid & 0.003699 & 0.633591 & 1.745718 \\
\hline TCA & 0.00393 & 0.248581 & 1.900476 \\
\hline Hydroxypropionic acid & 0.006305 & 0.510194 & 1.580288 \\
\hline Isovaleric acid & 0.010462 & 0.562201 & 1.265562 \\
\hline GHCA & 0.012991 & 0.43798 & 1.342739 \\
\hline GCDCA & 0.016901 & 0.333877 & 1.786831 \\
\hline Glycolic acid & 0.017797 & 0.773663 & 1.568525 \\
\hline Galactonic acid & 0.027913 & 0.486066 & 1.195792 \\
\hline Glucose & 0.027913 & 1.313563 & 1.386856 \\
\hline Tyrosine & 0.029297 & 1.43987 & 1.071764 \\
\hline Methylsuccinic acid & 0.029297 & 0.697323 & 1.928776 \\
\hline Ricinoleic acid & 0.029297 & 0.680282 & 1.278445 \\
\hline THCA & 0.042643 & 0.161662 & 1.658173 \\
\hline
\end{tabular}


Table S4 Four most significantly altered canonical pathways between BA and comparison infants.

\begin{tabular}{|l|l|l|l|l|l|}
\hline Metabolite & $\begin{array}{l}\text { Total_In_- } \\
\text { Pathway }\end{array}$ & Expected & Hits & Raw P & Enriched_Compounds \\
\hline $\begin{array}{l}\text { Valine, leucine } \\
\text { and isoleucine } \\
\text { biosynthesis }\end{array}$ & 0.078521 & 2 & 0.002459 & $\begin{array}{l}\text { alpha-Ketoisovaleric acid } \\
\text { Ketoleucine }\end{array}$ \\
\hline $\begin{array}{l}\text { Valine, leucine } \\
\text { and isoleucine } \\
\text { degradation }\end{array}$ & 0.11633 & 2 & 0.005366 & $\begin{array}{l}\text { alpha-Ketoisovaleric acid } \\
\text { Ketoleucine }\end{array}$ \\
\hline $\begin{array}{l}\text { Primary bile acid } \\
\text { biosynthesis }\end{array}$ & 47 & 0.13668 & 2 & 0.007364 & $\begin{array}{l}\text { GCA } \\
\text { TCA }\end{array}$ \\
\hline $\begin{array}{l}\text { Glutamine and } \\
\text { glutamate } \\
\text { metabolism }\end{array}$ & 11 & 0.03199 & 1 & 0.031594 & Glutamic acid \\
\hline
\end{tabular}


Table S5 The AUC, cutoff, sensitivity and specificity value of single altered metabolites

\begin{tabular}{|c|c|c|c|c|}
\hline Metabolite & Sensitivity & Specificity & Cutoff & AUC \\
\hline THCA & \begin{tabular}{|l|}
$0.89(81.17 \%$ \\
$94.38 \%)$
\end{tabular} & $\begin{array}{l}0.81(58.09 \% \\
94.55 \%) \\
\end{array}$ & 0.679 & $\begin{array}{ll}0.8519(0.7347 \text { to } \\
0.9692)\end{array}$ \\
\hline $\begin{array}{l}\text { 2-hydroxyglutaric } \\
\text { acid }\end{array}$ & $\begin{array}{l}0.67(56.88 \% \\
76.08 \%)\end{array}$ & $\begin{array}{l}0.81(58.09 \% \\
94.55 \%)\end{array}$ & 10.8 & $\begin{array}{l}0.7876(0.6654 \\
\text { to } 0.9098)\end{array}$ \\
\hline Ketoleucine & $\begin{array}{l}0.6(49.72 \% \\
69.67 \%)\end{array}$ & $\begin{array}{l}0.95(76.18 \% \\
99.88 \%)\end{array}$ & 15.75 & $\begin{array}{l}0.8271(0.7438 \text { to } \\
0.9105)\end{array}$ \\
\hline Indoleacetic acid & $\begin{array}{l}0.59(48.71 \% \\
68.74 \%) \\
\end{array}$ & $\begin{array}{l}0.86(63.66 \% \\
96.95 \%) \\
\end{array}$ & 1.14 & $\begin{array}{l}0.7738(0.6713 \\
\text { to } 0.8763)\end{array}$ \\
\hline $\begin{array}{l}\text { alpha-Ketoisovaleri } \\
\text { c acid }\end{array}$ & \begin{tabular}{ll|}
$0.78(68.61 \%$ & to \\
$85.67 \%)$ & \\
\end{tabular} & $\begin{array}{l}0.81(58.09 \% \\
94.55 \%)\end{array}$ & 1.08 & $\begin{array}{l}0.8086(0.7005 \\
\text { to } 0.9166)\end{array}$ \\
\hline Glutamic acid & $\begin{array}{l}0.84(75.32 \% \\
90.57 \%)\end{array}$ & $\begin{array}{ll}0.762(52.83 \% & \text { to } \\
91.78 \%) & \end{array}$ & 78.25 & $\begin{array}{l}0.8610(0.7690 \text { to } \\
0.9529)\end{array}$ \\
\hline GCA & $\begin{array}{l}0.9(82.38 \% \\
95.10 \%) \\
\end{array}$ & $\begin{array}{ll}0.67(43.03 \% & \text { to } \\
85.41 \%) & \\
\end{array}$ & 1.88 & $\begin{array}{ll}0.8243(0.7138 \text { to } \\
0.9348)\end{array}$ \\
\hline TCA & $\begin{array}{l}0.65(54.81 \% \text { to } \\
74.27 \%)\end{array}$ & $\begin{array}{l}0.76(52.83 \% \\
91.78 \%)\end{array}$ & 1.14 & $\begin{array}{l}0.8157(0.7192 \\
\text { to } 0.9122)\end{array}$ \\
\hline
\end{tabular}


Table S6 The AUC, cutoff, sensitivity and specificity value of altered metabolites combinations

\begin{tabular}{|c|c|c|c|c|}
\hline Metabolite & Sensitivity & Specificity & Cutoff & AUC \\
\hline $\begin{array}{l}\text { alpha-ketoisovaleric acid } \\
\text { THCA }\end{array}$ & $\begin{array}{c}0.7619 \\
(52.83 \% \text { to } \\
91.78 \%) \\
\end{array}$ & $\begin{array}{c}0.95(88.72 \% \text { to } \\
98.36 \%)\end{array}$ & -0.1190 & $\begin{array}{c}0.9205 \\
(0.8632- \\
0.9777)\end{array}$ \\
\hline $\begin{array}{l}\text { Indoleacetic-acid } \\
\text { 2-hydroxyglutaric acid } \\
\text { THCA }\end{array}$ & $\begin{array}{c}0.9048 \\
(69.62 \% \text { to } \\
98.83 \% \\
\end{array}$ & $\begin{array}{c}0.92 \\
(84.84 \% \text { to } \\
96.48 \%) \\
\end{array}$ & -0.3360 & $\begin{array}{c}0.9381(0.8736 \\
-1)\end{array}$ \\
\hline $\begin{array}{l}\text { Indoleacetic acid } \\
\text { Ketoleucine } \\
\text { 2-hydroxyglutaric acid } \\
\text { THCA }\end{array}$ & $\begin{array}{c}0.8571 \\
(63.66 \% \text { to } \\
96.95 \%\end{array}$ & $\begin{array}{c}0.95 \\
(88.72 \% \text { to } \\
98.36 \%\end{array}$ & -0.2415 & $\begin{array}{c}0.9443 \\
(0.8857-1)\end{array}$ \\
\hline $\begin{array}{l}\text { Indoleacetic acid } \\
\text { ketoleucine } \\
\text { 2-hydroxyglutaric acid } \\
\text { THCA } \\
\text { GCA }\end{array}$ & $\begin{array}{c}0.9048 \\
(69.62 \% \text { to } \\
98.83 \%)\end{array}$ & $\begin{array}{c}0.88 \\
(79.98 \% \text { to } \\
93.64 \%)\end{array}$ & -0.1697 & $\begin{array}{c}0.9448(0.885- \\
1)\end{array}$ \\
\hline $\begin{array}{l}\text { Indoleacetic acid } \\
\text { Ketoleucine } \\
\text { 2-hydroxyglutaric acid } \\
\text { THCA } \\
\text { TCA } \\
\text { GCA }\end{array}$ & $\begin{array}{c}0.8095 \\
(58.09 \% \text { to } \\
94.55 \%)\end{array}$ & $\begin{array}{c}0.97 \\
(91.48 \% \text { to } \\
99.38 \%)\end{array}$ & -0.2986 & $\begin{array}{c}0.941(0.8784- \\
1)\end{array}$ \\
\hline $\begin{array}{l}\text { Indoleacetic acid } \\
\text { alpha-ketoisovaleric acid } \\
\text { Ketoleucine } \\
\text { 2-hydroxyglutaric acid } \\
\text { THCA } \\
\text { TCA } \\
\text { GCA }\end{array}$ & $\begin{array}{c}0.8095 \\
(58.09 \% \text { to } \\
94.55 \%)\end{array}$ & $\begin{array}{c}0.98 \\
(92.96 \% \text { to } \\
99.76 \%)\end{array}$ & -0.3196 & $\begin{array}{c}0.939(0.8792- \\
0.9989)\end{array}$ \\
\hline $\begin{array}{l}\text { THCA } \\
\text { 2-hydroxyglutaric acid } \\
\text { Ketoleucine } \\
\text { Indoleacetic acid } \\
\text { alpha-Ketoisovaleric acid } \\
\text { Glutamic acid } \\
\text { GCA } \\
\text { TCA }\end{array}$ & $\begin{array}{c}0.91 \\
(83.60 \% \text { to } \\
95.80 \%)\end{array}$ & $\begin{array}{c}0.905(69.62 \% \text { to } \\
98.83 \%)\end{array}$ & -1.109 & $0.945(0.878,1)$ \\
\hline
\end{tabular}

